\title{
Shape-Guided Active Contour Based Segmentation and Tracking of Lumbar Vertebrae in Video Fluoroscopy Using Complex Wavelets
}

\author{
Alexander Wong ${ }^{1}$, Akshaya Mishra ${ }^{1}$, Paul Fieguth ${ }^{1}$, David Clausi ${ }^{1}$, Nadine M. Dunk ${ }^{2}$, Jack P. Callaghan ${ }^{2}$ \\ ${ }^{1}$ Department of Systems Design Engineering, ${ }^{2}$ Department of Kinesiology \\ University of Waterloo, Waterloo, Ontario, Canada
}

\begin{abstract}
This paper presents a novel shape-guided active contour based approach for segmenting and tracking lumbar vertebrae in video fluoroscopy using complex-valued wavelets. representations. Due to low radiation exposure levels, fluoroscopic images are characterized by low signal-to-noise ratios, low contrast resolution, and illumination non-homogeneities both spatially and temporally, making current methods illsuited for segmenting and tracking lumbar vertebrae based on existing energy functionals. Furthermore, current methods perform poorly in situations characterized by high curvature as found in the structure of lumbar spine vertebrae.

In this paper, a novel iterative estimation approach is used to determine an external energy functional based on complex wavelets. A shaped-guided algorithm is used to evolve the contour around a lumbar spine vertebra based on the complex wavelet energy. The high curvature exhibited by the lumbar spine vertebra is addressed through a novel importance sampling scheme. Experimental results show that the proposed algorithm achieves significantly better segmentation and tracking performance for lumbar spine vertebrae in fluoroscopic images when compared to existing techniques.
\end{abstract}

\section{INTRODUCTION}

Sedentary occupations that expose workers to prolonged sitting are associated with an increased risk of developing low back pain (LBP) [1], [2]. While the link between LBP and sitting has been attributed to the flexed curvature of the lumbar spine [3], the main causes are not well understood, thus making the condition difficult for clinicians to diagnosis. To improve the understanding of factors associated with LBP, it is necessary to quantify rhythms, delays and alterations in movement patterns related to LBP symptoms. Traditionally, the motion of the lumbar spine was acquired using standard radiographic systems. However, due to the relatively high level of radiation exposure of such systems, only a small number of extreme positions can be acquired, making a full analysis of continuous lumbar spine motion very difficult. A more recent approach that has been shown to be effective is the use of digital video fluoroscopy systems, which consist of an x-ray source, an image intensifier, and a video recorder. Due to the relatively low radiation exposure level of such systems, video fluoroscopy can be used to capture an entire sequence of the lumbar spine motion at a lower exposure level than a single image captured using standard radiographic systems. A typical fluoroscopic image of the lumbar-sacral region is shown in Fig. 1.

To aid in the analysis of lumbar spine motion, it is important to segment and track the motion of the individual

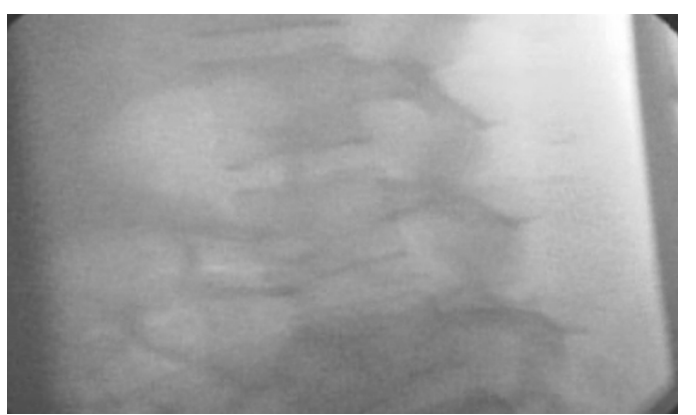

Fig. 1. A fluoroscopic image of the lumbar-sacral region. It can be observed that the image is contaminated by a high level of noise as well as exhibiting spatial illumination non-homogeneities and low contrast resolution.

lumbar spine vertebrae over a series of images over time. A number of techniques have been proposed for segmenting and tracking lumbar spine vertebrae in video fluoroscopy [4], [5], [6], [7], [8]. There are several drawbacks to existing methods that may hinder their effectiveness in non-ideal, real-world situations. Template matching based approaches to vertebrae tracking [4], [5], [6] are highly sensitive to geometric distortions, which often occur in fluoroscopic images since image intensifiers require electrons to be focused on an input screen with a curved surface. This issue was addressed by Wong et al. [7], [8] who use an active contour based approach to the problem. However, current active contour based methods [9], [10], [11] have several issues that make them difficult to implement for the purpose of vertebrae segmentation and tracking. First, the thickness of the patient affects the level of radiation hitting the image intensifier [12], thereby resulting in illumination non-homogeneities which are problematic as current methods are very sensitive to such variations. Second, the structure of lumbar spine vertebrae is characterized by high curvature points with which current methods perform poorly.

The main contribution of this paper is a shape-guided active contour based approach to lumbar spine vertebrae segmentation and tracking in fluoroscopic images using complex wavelets. The proposed method is highly robust to illumination, to contrast non-homogeneities, and to high curvature points, which are the key challenges to vertebrae segmentation and tracking in fluoroscopic images. In this paper, the proposed method is described in Section II, and experimental results using dynamic sagittal fluoroscopic videos of the lumbar region are presented in Section III. 


\section{PRoposed Method}

The proposed method takes an iterative approach to the problem of vertebrae segmentation and tracking in fluoroscopic images. A complex wavelet external energy functional is first estimated based on image characteristics. During each iteration, a locally optimal contour solution is found based on the estimated external energy, importance sampling is performed based on curvature, and a least squares estimation is performed to enforce the shape prior of the vertebrae.

\section{A. Background}

An active contour can be modeled as follows:

$$
\mathbf{c}(a)=[x(a), y(a)], a \in[0, l]
$$

where $a$ is the arclength and $l$ is the total contour length. The total energy functional of a contour can be defined as follows:

$$
E_{\text {total }}=\int_{0}^{l}(\underbrace{\alpha\left|\frac{\partial \mathbf{c}}{\mathrm{da}}\right|^{2}}_{\text {elastic }}+\underbrace{\beta\left|\frac{\partial^{2} \mathbf{c}}{\mathrm{da}^{2}}\right|^{2}}_{\text {rigid }}+\gamma E_{\text {ext }}(\mathbf{c})) d a
$$

where $\alpha$ and $\beta$ are relaxation factors for the internal elasticity and rigidity constraints respectively, $E_{\text {ext }}$ is the external energy functional, $\gamma$ is the relaxation factor for the external energy functional. The optimal contour solution is determined by minimizing $E_{\text {total }}$.

\section{B. External Energy Functional Estimation}

In current active contour based methods, $E_{\text {ext }}$ is defined as the negative intensity gradient $(-\nabla I)$. However, this is unsuitable for the case of fluoroscopic images, which are characterized by low illumination and contrast nonhomogeneities. An external energy functional that addresses such issues can be obtained as the negative complex wavelet phase coherence moments [13]. However, it is difficult to extract useful complex wavelet phase coherence moments in situations characterized by low signal-to-noise ratios such as those found in fluoroscopic images. To address issues associated with noise, we utilize a noise-resilient approach to complex wavelet phase coherence moment estimation [14] to compute the external energy functional. The proposed approach can be described in detail as follows. Given an image $I_{0}$, the initial local phase coherence estimate $P_{0}$ is obtained at iteration $t=0$. At each iteration $t$ of the proposed estimation approach, the maximum complex wavelet phase coherence moments $\varpi_{t}$ is computed based on $P_{t-1}$. Based on $\varpi_{t}$, a new estimate of the image $I_{t}$ is computed using a moment-adaptive bilateral estimation approach:

$$
I_{t}(\underline{x})=\frac{\sum_{\psi} w\left(\underline{x}, \psi, \varpi_{t}(\underline{x})\right) I_{t-1}(\psi)}{\sum_{\psi} w\left(\underline{x}, \psi, \varpi_{t}(\underline{x})\right)}
$$

where the estimation weight $w$ is defined as the product of spatial and amplitudinal weighing functions $w_{s}$ and $w_{a}$ over a local neighbborhood $\psi$ :

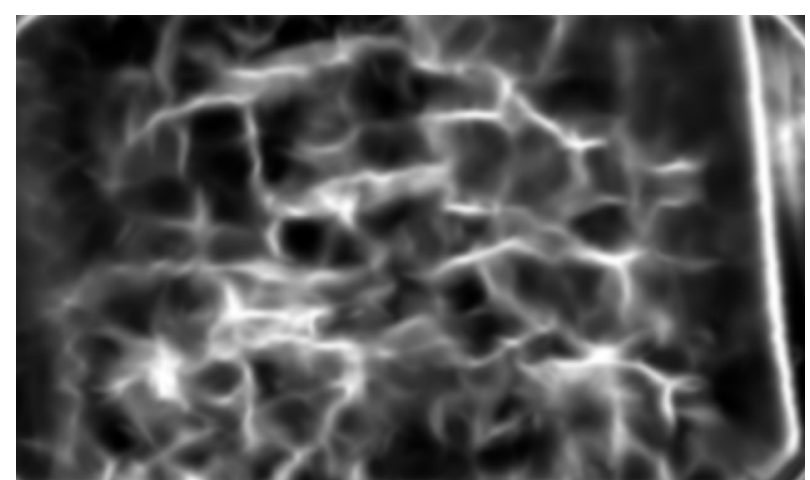

Fig. 2. Estimated complex wavelet phase coherence moments for the fluoroscopic image shown in Fig. 1. It can be observed that strong structural information are clearly accentuated, which is important for the purpose of segmentation.

$$
\begin{gathered}
w\left(\underline{x}, \psi, \varpi_{t}(\underline{x})\right)=w_{a}\left(\underline{x}, \psi, \varpi_{t}(\underline{x})\right) w_{s}\left(\underline{x}, \psi, \varpi_{t}(\underline{x})\right) \\
w_{s}\left(\underline{x}, \psi, \varpi_{t}(\underline{x})\right)=e^{-\frac{1}{2}\left(\frac{\|\underline{x}-\psi\|}{\sigma_{s}\left(\varpi_{t}(\underline{x})\right)}\right)^{2}} \\
w_{a}\left(\underline{x}, \psi, \varpi_{t}(\underline{x})\right)=e^{-\frac{1}{2}\left(\frac{\|I(\underline{x})-I(\psi)\|}{\sigma_{a}\left(\varpi_{t}(\underline{x})\right)}\right)^{2}}
\end{gathered}
$$

The estimated $I_{t}$ is then used to estimate the local phase coherence for the next iteration $P_{t+1}$. This process is repeated until the desired number of iterations is completed to obtain the final moment estimate $\tilde{\varpi}$. The estimated complex wavelet phase coherence moments using the proposed method for a fluoroscopic image is shown in Fig. 2. The external energy functional can then be defined as follows:

$$
E_{e x t}=-\tilde{\varpi}
$$

\section{Shape-Guided Active Contour Evolution}

To evolve the active contour at each iteration, the problem is formulated as a Hidden Markov Model (HMM) and the local optimum solution is found using the Viterbi algorithm [15], [16] based on the estimated complex wavelet energy functional. The active contour is modeled using $q$ discrete points and a search is performed for the moment normals to the contour at each of the $q$ points with $p$ discrete nodes distributed along each normal. This results in a total of $p^{q}$ possible solutions. Based on the external energy $E_{\text {ext }}=$ $-\tilde{\varpi}$ at each node, the Viterbi algorithm determines the partial probability at each node and determines the best sequence of states that maximizes probability along the contour. Based on this sequence, the evolved contour can be found.

The evolved contour found using the Viterbi algorithm is typically insufficient due partly to the lack of prior constraints. Most current methods deal with this issue by asserting first or second order constraints. However, it is problematic for segmenting lumbar spine vertebrae since the structure of the vertebrae is characterized by high curvature 
regions. In the proposed method, the problem of high curvature points is addressed by generating points along the active contour using a novel curvature-adaptive importance sampling scheme. The density of points along the contour is adapted in proportional to the absolute value of curvature such that more points are located at high curvature regions. Upper and lower bounds for point density is enforced to prevent oversampling high curvature regions or undersampling low curvature regions.

As stated earlier, it is necessary to impose prior constraints to obtain the desired contour around the lumbar spine vertebrae. However, it is very difficult to integrate prior constraints into the Viterbi algorithm. The proposed algorithm works around this issue by first performing the Viterbi algorithm without prior constraints and then fusing prior constraints with the estimated complex wavelet phase coherence moments using constrained least squares optimization. The fusion process can be defined as follows:

$$
\hat{Z}=\left(C^{T} R^{-1} C+P^{-1}\right)^{-1} C^{T} R^{-1}\left(\underline{\Omega}-\underline{\mu}_{t}\right)
$$

where $P$ is the prior constraints, $C$ is the state matrix, $\underline{\Omega}$ is the estimated moments after dynamic programming, $\underline{\mu}_{t}$ is the translation vector computed from moment differences of consecutive frames, and $R$ are the measurement weights for each point based on estimated moments. The resulting contour is then refined over multiple iterations.

\section{EXPERIMENTAL RESULTS}

To evaluate the effectiveness of the proposed algorithm, a vertebra in the lumbar-sacral region of the spine was segmented and tracked over time in two test sagittal fluoroscopic videos. A summary of the test sets is shown below:

- BHN: male (age $=22$; height $=1.80 \mathrm{~m}$; mass $=77.6 \mathrm{~kg}$ )

- AUR: female (age=24; height $=1.57 \mathrm{~m}$; mass $=45.8 \mathrm{~kg}$ )

Both test videos consisted of nine frames depicting the motion of the spine going from an upright seated position to a slouched seated position. The test videos were obtained using a Siemens Siremobil Compact fluoroscopic imaging system equipped with a 9-inch image intensifier with average $\mathrm{x}$-ray technique factors of $3.4 \mathrm{~mA}$ and $107 \mathrm{kV}$.

The test videos are representative of non-ideal, real-world scenarios, where the fluoroscopic images are characterized by low signal-to-noise ratios as well as illumination and contrast non-homogeneities both spatially and temporally. The gradient vector flow (GVF) method [10] was also evaluated for comparison purposes. It is important to note that the GVF method was slower than the proposed method by over a factor of 20. It is also important to note that the GVF method fails to track the vertebrae beyond the initial frame for both test cases. Therefore, to make the GVF method function reasonable for comparison purposes, the contour was re-initialized after each frame. For the proposed method, the contour was initialized solely for the first frame, which is important for the purpose of tracking since it is impractical to re-initialize the contour for longer sequences. The normalized MSE between the ground truth contour and

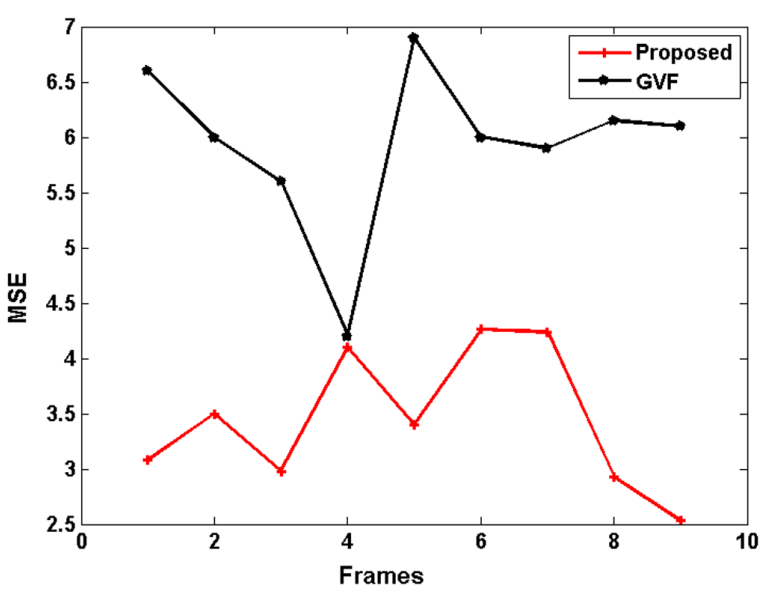

(a) AUR

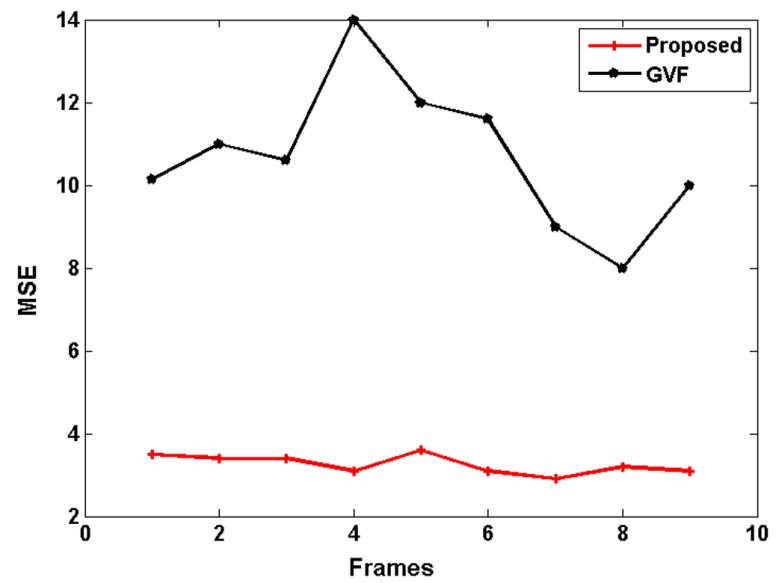

(b) $\mathrm{BNH}$

Fig. 3. Normalized MSE results for AUR and BNH test videos. It can be observed that the proposed method achieves noticeably lower MSE than the gradient vector flow (GVF) method for all frames for both test videos.

the obtained contours using GVF and the proposed method is computed over all frames within the test videos. The MSE results for the AUR and BNH sequences are shown in Fig. 3. In the both cases, the proposed method performed significantly better than the GVF method. Sample tracking results for the AUR and BNH sequences are shown in Fig. 4 and Fig. 5 respectively. The performance improvements of the proposed method over GVF were particularly evident in the BHN case, where the contours determined by the GVF method were significantly deformed and offset when compared to the actual vertebrae. The proposed method was able to segment and track the target vertebra over the full sequence in both test videos with a high level of accuracy. This demonstrates the effectiveness of the proposed method in segmenting and tracking lumbar spine vertebrae over time in fluoroscopic images. 


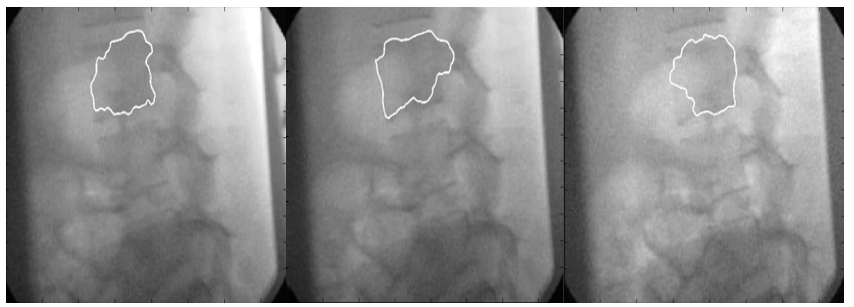

(a) GVF method (with re-initialization)

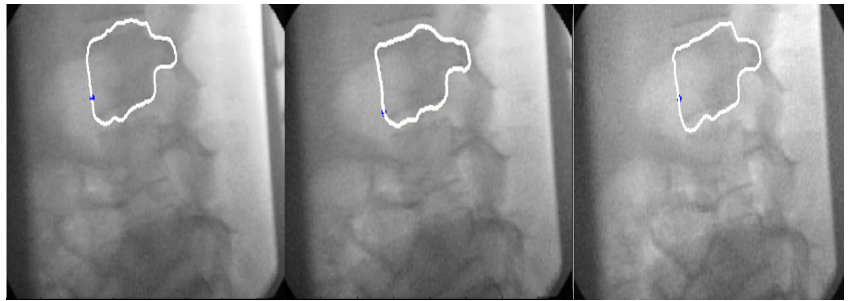

(b) Proposed method

Fig. 4. AUR tracking results for frames 153, 156, and 160. It can be observed that the proposed method performs noticeably better at segmenting and tracking the vertebra compared to the GVF method without the need for re-initialization.

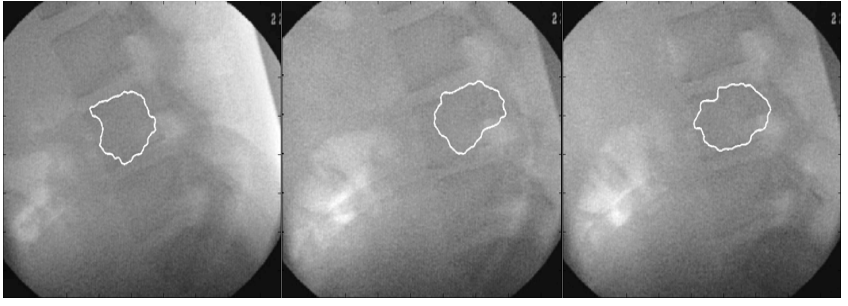

(a) GVF method (with re-initialization)

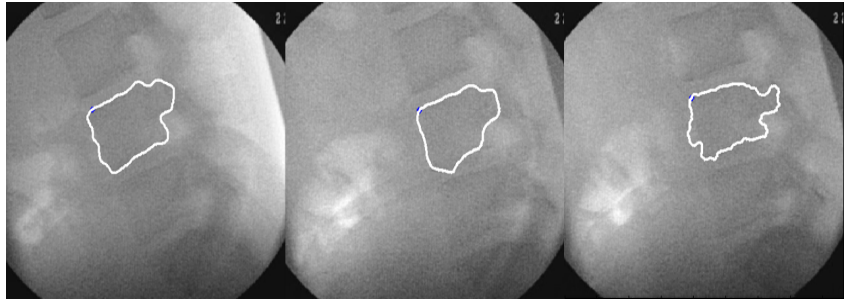

(b) Proposed method

Fig. 5. BNH tracking results for frames 1,4 , and 8 . It can be observed that the proposed method performs substantially better than the GVF method, which is unable to segment and track the vertebra at all.

\section{CONClusions And Future Work}

In this paper, we have introduced a novel approach to the problem of lumbar spine vertebrae segmentation and tracking in fluoroscopic images. An iterative scheme for estimating complex wavelet moments was used to generate moments for the external energy functional, thereby addressing the issues of contrast and illumination non-homogeneities and low signal-to-noise ratios. A dynamic programming approach was used to provide fast initial contour evolution. Issues associated with high curvature points in lumbar spine vertebrae structure were addressed through the use of a novel importance sampling scheme. Experimental results showed that good segmentation and tracking accuracy of lumbar spine vertebrae can be achieved for fluoroscopic image sequences compared to existing techniques. Future work involves extracting detailed motion information about the vertebrae from the tracked contour for the purpose of quantifying alterations in movement patterns related to LBP symptoms in order to aid clinical diagnosis.

\section{ACKNOWLEDGMENT}

The authors would like to thank the Natural Sciences and Engineering Research Council (NSERC) of Canada for funding this project. The authors would also like to thank the Vision and Image Processing Group at the University of Waterloo.

\section{REFERENCES}

[1] A. Magora, "Investigations of the relation between low back pain and occupation," Industrial Medicine, vol. 41, no. 12, pp. 5-9, 1972.

[2] J. Frymoyer, M. Pope, M. Costanza, J. Rosen, J. Goggin, and D. Wilder, "Epidemiologic studies of low back pain," Spine, vol. 5 pp. 419-423, 1980.
[3] D. Wilder and M. Pope, "Epidemiological and aetiological aspects of low back pain in vibration environments - an update," Clinical Biomechanics, vol. 11, no. 2, pp. 61-73, 1996.

[4] L. Penning, R. Irwan, and M. Oudkerk, "Measurement of angular and linear segmental lumber spine flexion-extension motion by means of image registration,” European Spine Journal, Vol. 14, pp. 163-170, 2005.

[5] P. Bifulco, M. Cesarelli, R. Allen, M. Sansone, and M. Bracale, "Automatic recognition of vertebral landmarks in fluoroscopic sequences for analysis of intervertebral kinematics," Medical \& Biological Engineering \& Computing, vol. 39, pp. 65-75, 2001.

[6] J. Muggleton and R. Allen, "Automatic location of vertebrae in digitized videofluoroscopic images of the lumbar spine", Med. Eng. Phys., vol. 19, no. 1, pp. 77-89, 1997.

[7] S. Wong, K. Wong, W. Wong, C. Leong, and D. Luk, "Tracking Lumbar Vertebrae in Digital Videofluoroscopic Video Automatically", Proc. MIAR 2004, LNCS 3150, pp. 154-162, 2004.

[8] W. Wong, D. Luk, C. Leong, F. Wong, and K. Wong, "Continous Dynamic Spinal Motion Analysis", Spine, vol. 31, no. 4, pp. 414-419, 2006.

[9] M. Kass, A. Witkin, and D. Terzopoulo, "Snakes: Active Contour Models", IJCV, vol. 1, no. 4, pp. 321-331, 1988.

[10] C. Xu and J. Prince, "Snakes, Shapes, and Gradient Vector Flow", IEEE Trans. on Image Processing., vol. 7, no. 3, pp. 359-369, 1998.

[11] C. Li, C. Xu, C, Gui, and M. Fox, "Level set evolution without reinitialization: a new variational formulation," Proc. IEEE Computer Society Conference on Computer Vision and Pattern Recognition, vol. 1, pp. 430-436, 2005.

[12] J. Bushberg, J. Seibert, E. Leidholdt Jr., and J. Boone, Essential physics of medical imaging, Williams \& Wilkins, 2002.

[13] P. Kovesi, "Phase congruency detects corners and edges," Proc. Australian Pattern Recognition Society Conference, pp. 309-318, 2003.

[14] A. Wong, "An iterative approach to improved local phase coherence estimation", Proc. CRV 2008, 2008.

[15] A. Viterbi, "Error bounds for convolutional codes and an asymptotically optimum decoding algorithm", IEEE Trans. on Information Theory, IT-13, pp. 260-269, 1967.

[16] A. Mishra, P. Fieguth, and D. Clausi, "Accurate boundary localization using dynamic programming on snake", Proc. CRV 2008, 2008. 